\title{
Multipole modes and spin features in the Raman spectrum of nanoscopic quantum rings
}

\author{
Agustí Emperador, Martí Pi, and Manuel Barranco \\ Departament ECM, Facultat de Física, Universitat de Barcelona, E-08028 Barcelona, Spain \\ Enrico Lipparini \\ Dipartimento di Fisica, Università di Trento, and INFM sezione di Trento, I-38050 Povo, Italy
}

(Received 15 March 2001; published 18 September 2001)

\begin{abstract}
We present a systematic study of ground state and spectroscopic properties of many-electron nanoscopic quantum rings. Addition energies at zero magnetic field $(B)$ and electrochemical potentials as a function of $B$ are given for a ring hosting up to 24 electrons. We find discontinuities in the excitation energies of multipole spin and charge density modes, and a coupling between the charge and spin density responses that allow to identify the formation of ferromagnetic ground states in narrow magnetic field regions. These effects can be observed in Raman experiments, and are related to the fractional Aharonov-Bohm oscillations of the energy and of the persistent current in the ring.
\end{abstract}

DOI: 10.1103/PhysRevB.64.155304

PACS number(s): 73.21.-b, 85.35.Be

\section{INTRODUCTION}

Very recently, quantum rings in InAs-GaAs heterostructures have been fabricated in the nanometer scale, ${ }^{1-3}$ and the capacitance and far-infrared (FIR) response have been measured for the one- and two-electron quantum rings. Previously, the FIR response ${ }^{4}$ had been measured for mesoscopic rings in GaAs- $\mathrm{Ga}_{x} \mathrm{Al}_{1-x} \mathrm{As}$ heterostructures, for which a description based on classical and hydrodynamical models works fairly well. ${ }^{5,6}$ In nanoscopic rings quantum effects are important and for this reason theoretical studies at a more microscopic level have been undertaken. ${ }^{7-19}$

In some of these calculations a major emphasis has been put on describing the Aharonov-Bohm (AB) quantum effect which manifests in the presence of an external magnetic field $(B)$ as the existence of a persistent current, and leads to periodic oscillations in the energy spectrum and the persistent current as a function of $B$. These $\mathrm{AB}$ oscillations have been observed in mesoscopic rings in a GaAlAs/GaAs heterostructure by measuring the conductance across the ring. ${ }^{20}$

The experimental results for one- and two-electron nanoscopic rings have been theoretically analyzed ${ }^{21}$ using the current density (CDFT) and time-dependent local-spin densityfunctional (TDLSDFT) theories, and a good agreement between theory and experiment is found (see also Refs. 16 and 19). Motivated by this success, in this work we extend this approach to a systematic study of ground state (g.s.) and spectroscopic properties of nanoscopic rings containing up to $N=24$ electrons. Our aim is to show the physical appearance of quantities that could be measured in the future if the problem of fabricating many-electron nanoscopic rings is eventually solved. Specifically, we have obtained electrochemical potentials $\mu(N)=E(N)-E(N-1)$, where $E(N)$ is the total energy of the $N$-electron ring, addition energies $\Delta_{2}(N)$ $=\mu(N+1)-\mu(N)=E(N+1)-2 E(N)+E(N-1)$, and the spin and charge density responses at finite on-plane transferred wave vector, which are relevant for the analysis of Raman spectra. All these quantities have been measured in $\mathrm{N}$-electron quantum dots, see, for example, Refs. 22-27, and references therein. Particular emphasis is given to spectro- scopic results. We show that the collective excitation energies exhibit discontinuities in their $B$ dependence, which are a manifestation of changes in the spin configuration of the ground state, and that in a ring-wire would be related to the fractional Aharonov-Bohm effect.

The structure of the ring gs has been obtained within the CDFT as described in Refs. 28 and 29. To obtain the charge and spin density responses we have used TDLSDFT as described in Refs. 30 and 31. Essentially, the method implies that to obtain the ground state and excited modes of the system, besides the direct electron-electron interaction, exchange and correlation effects have been included in a local density approximation. We refer the reader to these references for a comprehensive exposure of the CDFT and TDLSDFT, of direct applicability here changing the shape of the confining potential from a dotlike potential to a ringlike potential.

\section{GROUND STATE RESULTS}

Following Ref. 7, we have modeled the ring confining potential by a parabola

$$
V^{+}(r)=\frac{1}{2} m \omega_{0}^{2}\left(r-R_{0}\right)^{2}
$$

with $R_{0}=20 \mathrm{~nm}$ and $\omega_{0}=12 \mathrm{meV}$. These values are close to the ones used ${ }^{21}$ to describe the rings studied by Lorke et al. ${ }^{3}$ The electron effective mass $m^{*}=0.063$ (we write $m$ $=m^{*} m_{e}$ with $m_{e}$ being the physical electron mass) and effective gyromagnetic factor $g^{*}=-0.43$ have been taken from the experiments, ${ }^{32-34}$ and the value of the dielectric constant has been taken to be $\epsilon=12.4$. The model is strictly two-dimensional, and as a consequence of circular symmetry, the single particle (s.p.) wave functions are eigenstates of the orbital angular momentum $l_{z}$ and can be written as $u_{n l \sigma}(r) e^{-i l \theta}$ with $l=0, \pm 1, \pm 2, \ldots$. Notice that with this convention, the s.p. orbital angular momentum is $-l$.

Some times we have used effective atomic units, defined by $\hbar=e^{2} / \epsilon=m=1$. In this system of units, the length unit is 


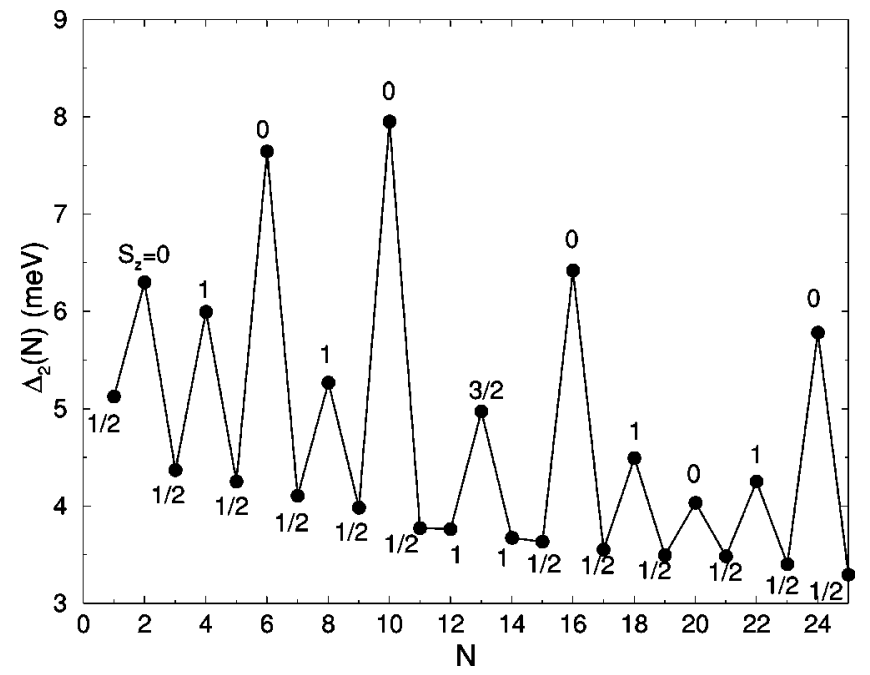

FIG. 1. Addition energies $\Delta_{2}(N)(\mathrm{meV})$ at $B=0$ as a function of the number of electrons in the ring.

the effective Bohr radius $a_{0}^{*}=a_{0} \epsilon / m^{*}$, and the energy unit is the effective Hartree $H^{*}=H m^{*} / \epsilon^{2}$. This yields $a_{0}^{*}$ $\sim 10.4 \mathrm{~nm}$ and $H^{*} \sim 11.15 \mathrm{meV}$. The Bohr magneton is defined as $\mu_{B}=\hbar e / 2 m_{e} c$.

Figure 1 shows the addition energies $\Delta_{2}(N)$ at zero magnetic field. Saw-tooth structures and large peaks at magic numbers $N=2,6,10,16$, and 24 that correspond to closeshell rings are clearly seen. Had we not taken into account electron-electron interactions (single-particle picture), the magic numbers would have been $N=2,6,10,16,20$, and 24 . The effect of interaction is to weaken some shell closures, for example, that at $N=20$ in the displayed results. The totalspin third-component of the g.s. configuration is indicated in the figure, and shows that Hund's first rule, according to which as degenerate states are filled, the total spin $S$ takes the maximum allowed value by the exclusion principle and becomes zero for closed shells, is satisfied up to $N=16$. For larger electron numbers Hund's first rule is violated and the higher spin state no longer is the g.s. In particular, $S_{z}$ becomes zero at $N=20$ instead of having the Hund's first rule value $S_{z}=2$. These considerations may change if the geometrical characteristics of the studied ring are different. In this respect, we want to point out that according to the one-dimensionality criterium established in Ref. 13, the rings we describe cannot be considered as ring wires, an obvious statement if one looks at the electron density plotted in Fig. 2.

Similarly to what happens in quantum dots, ${ }^{35}$ the application of an external magnetic field produces a nontrivial change in the ring structure, inducing changes in the spin of the g.s. configuration. This can be seen in the middle panel of Fig. 3 where we have plotted the $B$ evolution of the sp energies up to $B=6.5 \mathrm{~T}$ for $N=10$. Paying attention to the 4.8 $<B<6$ T region for example, one may observe a level crossing around the Fermi energy when $B \approx 5 \mathrm{~T}$. An electron moves from the $(0, \downarrow)$ to the $(5, \uparrow)$ s.p. level and, as a consequence, the spin state changes from a singlet to a triplet state (ST transition). In such a way the system minimizes its exchange energy and the gs becomes ferromagnetic. A further

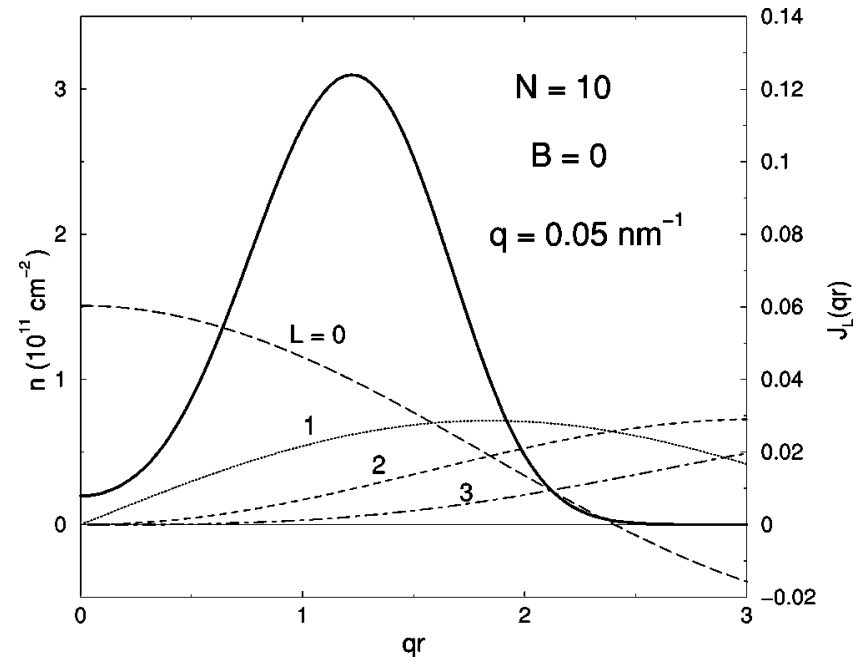

FIG. 2. Electron density (solid line) for the $N=10$ ring at $B$ $=0$. The dimensionless horizontal scale may be transformed into a conventional one recalling that $q=0.05 \mathrm{~nm}^{-1}$. The value of $J_{L}(q r)$ for $L=0$ to 3 is also shown (right scale).

increase of $B$ changes again the situation, and at $B \approx 5.8 \mathrm{~T}$ an electron moves now from the $(0, \uparrow)$ to the $(5, \downarrow)$ s.p. level and the system undergoes a triplet $\rightarrow$ singlet (TS) transition. Exchange gaps in the s.p. energies similar to this one also occur near $B \sim 1$ and $3 \mathrm{~T}$, and again at $B \approx 8.5 \mathrm{~T}$ (not shown in the figure), and are characteristic of even- $N$ rings before full magnetization. Exchange gaps have been analyzed in quantum dots to experimentally determine the contribution form the direct Coulomb and exchange electron-electron interaction to the total energy. ${ }^{36}$

The situation is quite different for odd- $N$ rings, for which the system always remains ferromagnetic $\left(S_{z} \neq 0\right)$. This can be seen in the top panel of Fig. 4, where we have plotted the $B$ evolution of the s.p. levels of the $N=9$ ring up to $B$ $=6.5 \mathrm{~T}$. Now, the level crossings around the Fermi energy occur without spin changing. The situation shown in this figure is similar for all the odd- $N$ rings considered in this work.

Level crossings strongly influence the $B$ behavior of the electrochemical potential, which is shown in Fig. 5 for 0 $\leqslant B \leqslant 10$ T up to $N=12$. For even electron numbers the number of ST transitions depends on $N$ and increases with it, as the number of level crossings to arrive at full polarization also increases. The critical $B$ value at which the system is fully polarized also increases with $N$, and is well visible in this figure up to $N=9$. For odd electron numbers, the spin of the gs configuration remains constant $\left(S_{z}=\frac{1}{2}\right)$ in a wide $B$ range, and from a certain $B$ value on it starts increasing up to full polarization.

The pairing between peaks in the electrochemical potential corresponding to consecutive $N$ values, such as, for example, 9 and 10, indicates antiparallel spin filling of a s.p. $l$ orbital by two electrons. This pairing is violated at $B=0$ for $N=4$ and 8 , and it is a manifestation of Hund's first rule. It is also violated in the narrow $B$ regions where the spin triplet state is the g.s. configuration of even- $N$ rings. This effect cannot be seen in Fig. 5 because of the $\Delta B=0.5$ T step used 


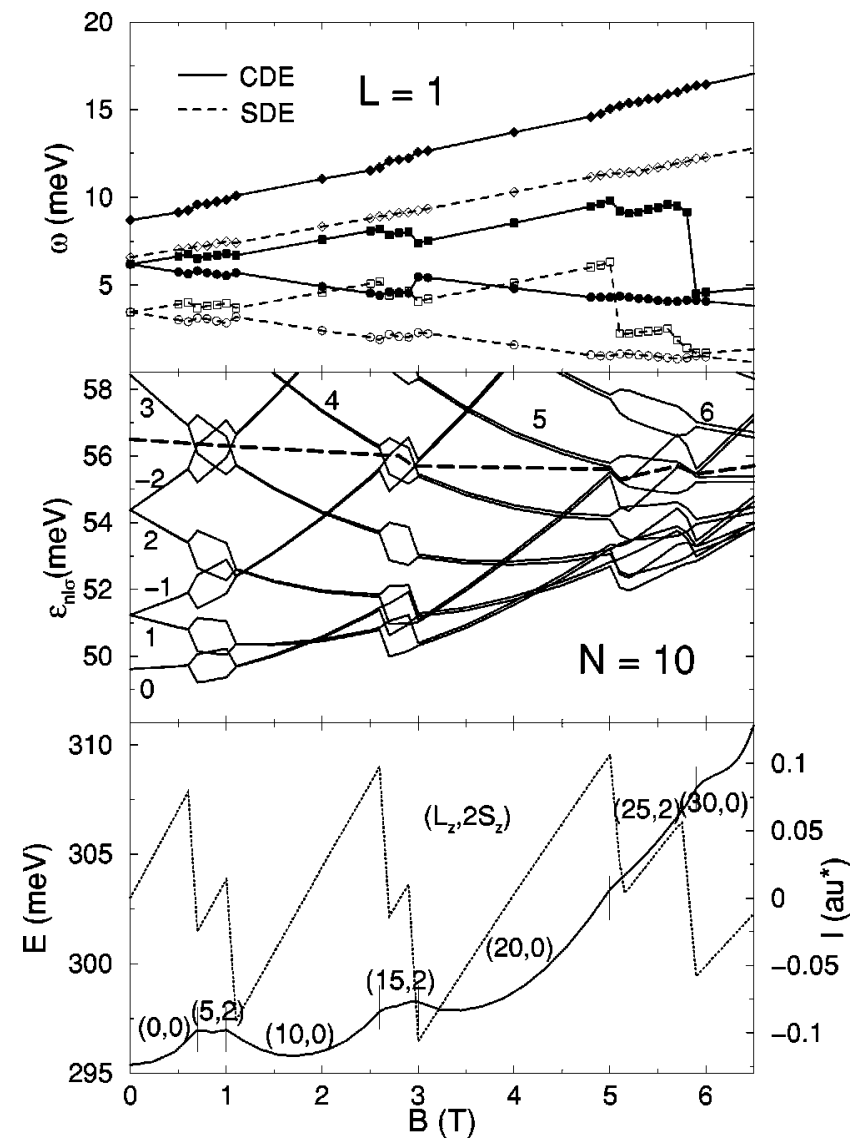

FIG. 3. Some results for the $N=10$ ring as a function of $B$. Top panel: dipole CDE's (filled symbols, solid lines) and SDE's (empty symbols, dashed lines) in the long-wavelength limit. The circles represent outer edge modes, and the squares inner edge modes. Diamonds: bulk modes. Middle panel: s.p. level energies. The $l$ value is indicated, and for each of them, the lower energy level corresponds to the spin-up orbital. The dashed line marks the boundary between occupied and empty states. Bottom panel: total energy (solid line, left scale), and persistent current (dotted line, right scale in effective atomic units). The values of the total spin and orbital angular momentum of the different g.s. phases are also indicated.

to obtain it, but can be seen in the top panel of Fig. 11 below, which gives a magnified view of $\mu(10)$ in the region around $B \sim 3 \mathrm{~T}$. This is the same behavior found for quantum dots in Ref. 36.

Quasiperiodic $B$ oscillations can be observed in the electrochemical potential. The changes in spin configurations and the maxima and minima of the electrochemical potential curves are related to s.p. level crossing at the Fermi energy. For example, for $N=10$ the $\mathrm{ST}$ transitions around $B=1,3$, and $5.5 \mathrm{~T}$, and the maxima of $\mu(10)$, can be related to the level crossings at these $B$ values shown in Fig. 3. The minima of $\mu(9)$ and $\mu(10)$ at $B \sim 2$ and $4.5 \mathrm{~T}$ correspond to the level crossings without spin change shown in Figs. 3 and 4.

These quasiperiodic oscillations are absent in quantum dots, ${ }^{24}$ and are a characteristic inherent to quantum rings. We recall that for a one-electron ring-wire of radius $R_{0}$ the energies are given by

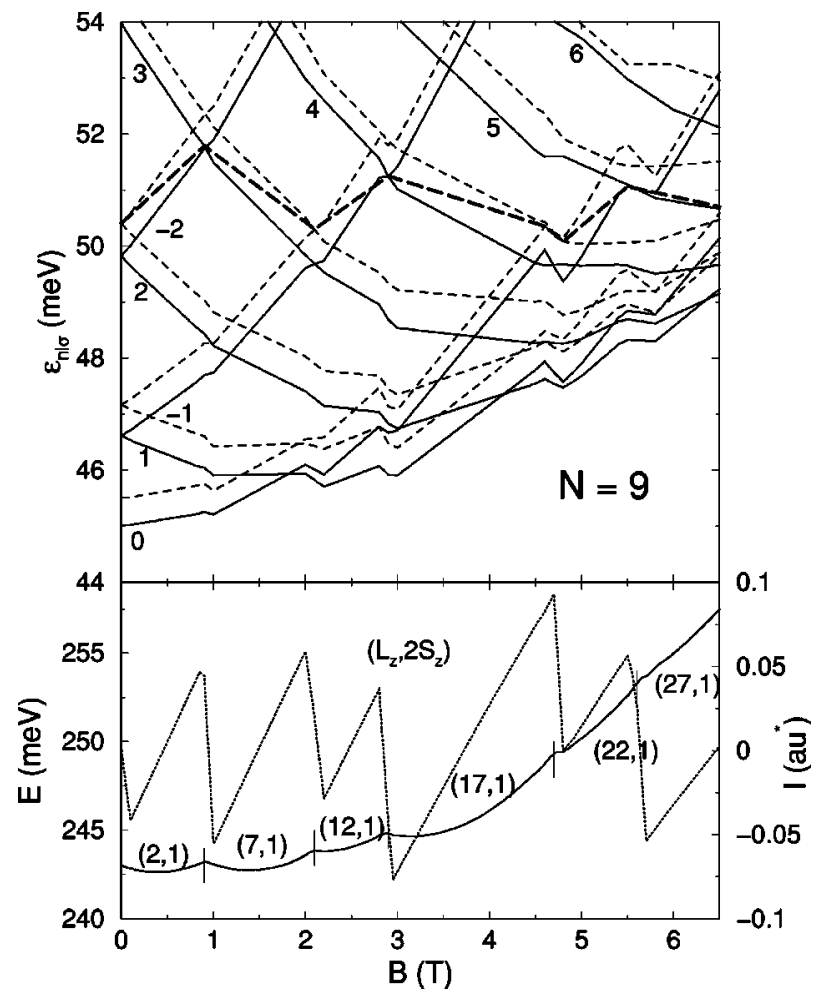

FIG. 4. Referring to the $N=9$ ring, we display as a function of $B$ : Top panel, the sp level energies. The thick dashed line marks the boundary between occupied and empty states. Spin-up s.p. orbitals are denoted by solid lines, and spin-down s.p. orbitals by dashed lines. Bottom panel: total energy (solid line, left scale) and persistent current (dotted line, right scale in effective atomic units). The values of the total spin and orbital angular momentum of the different g.s. phases are also indicated.

$$
\epsilon_{l}=\frac{\hbar^{2}}{2 m^{*} R_{0}^{2}}\left(l-\frac{\Phi}{\Phi_{0}}\right)^{2},
$$

where $\Phi / \Phi_{0}$ is the number of flux quanta penetrating the ring, $\Phi_{0}=h c / e$, and are periodic in the flux $\Phi=\pi R_{0}^{2} B$ with periodicity $\Phi_{0}$. The persistent current $I_{l}$ associated with the $l$ state

$$
I_{l}=-c \frac{\partial \epsilon_{l}}{\partial \Phi}
$$

has the same periodicity. As $B$ increases the occupied $l$ state changes and gives rise to discontinuities in the current $I_{l}$ (the $A B$ effect), which have been experimentally observed in a mesoscopic loop in a GaAs heterojunction. ${ }^{20}$

We have plotted in Figs. 3 and 4 the total energy $E$ and the persistent current determined as $I=\int J(r) d r$ with

$$
\mathbf{J}(\mathbf{r})=\mathbf{J}_{p}(r)-\frac{e}{m^{*} c} n(r) \mathbf{A}(r),
$$

where $\mathbf{J}_{p}(r)=-\hat{e}_{\theta} \sum l\left[u_{n l \sigma}(r)\right]^{2} / r$, being $\hat{e}_{\theta}$ the azimuthal vector unit, is the paramagnetic current that for manyelectron rings is obtained adding the s.p. paramagnetic currents, ${ }^{37}$ and the second term is the diamagnetic current, 


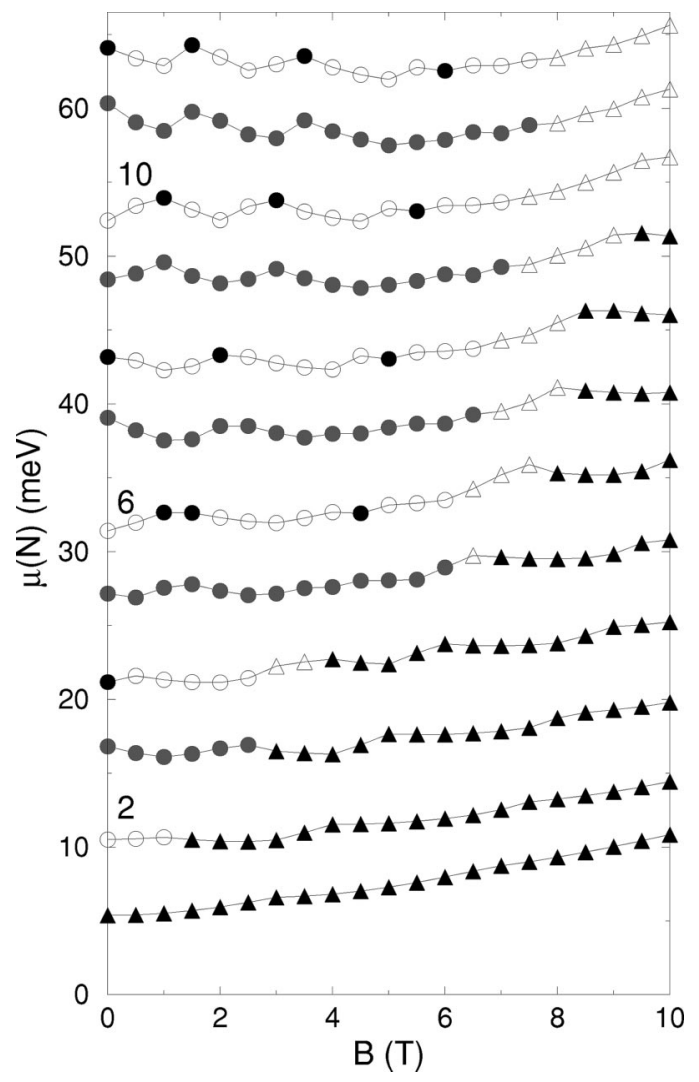

FIG. 5. Electrochemical potentials $\mu(N)=E(N)-E(N-1)$ as a function of $B$. Different symbols are used to distinguish g.s.'s with different $S_{z}$ values. There can be seen paramagnetic regions with $S_{z}=0$ (open circles), and a full spin-polarized region with $S_{z}$ $=N / 2$ (black triangles). Other gs's with a spin value $S_{z}=\frac{1}{2}$ (gray circles) and $S_{z}=1$ (black circles) may be identified. The region of steadily increasing polarization has been indicated by open triangles. The lines have been drawn to guide the eye.

being $n(r)=\Sigma\left[u_{n l \sigma}(r)\right]^{2}$ and $\mathbf{A}(r)$ the electron density and external vector potential, respectively.

In a many-electron nanoscopic ring-wire, electronelectron interactions give rise to changes in the gs configuration that decreases the period of the $\mathrm{AB}$ oscillations. ${ }^{38}$ This effect has been explained in Ref. 11 as a consequence of Hund's first rule which favors the occurence of ferromagnetic $S_{z}=1$ phases. A ring with a low degree of one dimensionality tends to lose the periodic features of the ideal ring wire, being somehow an intermediate case between a ring wire and a quantum dot, and for these systems the effect is not so marked, ${ }^{21}$ appearing as a small amplitude oscillation whose $B$ width coincides with that of the ferromagnetic phase (see Fig. 3). The crucial role played by the degree of one-dimensionality in the appearance of the $\mathrm{AB}$ oscillations can also be appraised comparing the results for two and four electron rings obtained in Refs. 11,13. In the case of an $S_{z}$ $=0$ ring as for $N=10$, we have found a period halving. For an $S_{z}=\frac{1}{2}$ ring similar to that with $N=9$, the oscillations due to ST and TS transitions are absent but there still is a period halving which in this case can be traced back to that arising for noninteracting spin- $\frac{1}{2}$ fermions. ${ }^{11,38}$ We have estimated that to have $\Phi=\Phi_{0}$ for a ring wire of radius equal to the maximum electronic density radius in Fig. 2 , one needs $B$ $\sim 2.2 \mathrm{~T}$. Consequently, a period of $\Phi_{0} / 2$ corresponds to a period of $B \sim 1 \mathrm{~T}$ as shown in the bottom panel of Fig. 4. A period $\sim \Phi_{0}$, i.e., $B \sim 2 \mathrm{~T}$ between large amplitude oscillations of the current shown in the bottom panel of Fig. 3 is also consistent with this picture.

We have also checked that calculating the current from the $B$ derivative of the total energy, as suggested by Eq. (3), one obtains discontinuities at the same $B$ values. However, both ways of calculating the persistent current do not yield the same value. This difference is attributed to the fact that our ring is not in a "clean" $\mathrm{AB}$ situation in which electrons move essentially in a $B$-free region. ${ }^{13}$ Rather, an appreciable magnetic flux is going across the effective ring surface.

\section{RAMAN SPECTRA}

Raman spectra are obtained through two-photon inelastic light scattering. The process consists in creating an electronhole pair (absorption of the incident photon) and subsequent recombination between valence and conduction bands (emission of the final photon). In this process ${ }^{39,40}$ the electrons of the conduction band are excited via the transfer of energy $\omega$ and momentum $q$. In a back scattering geometry, with incoming and scattered photon polarization vectors $\hat{\mathbf{e}}_{i}$ and $\hat{\mathbf{e}}_{s}$ lying in the $x-y$ plane perpendicular to $B$, the charge and spin density Raman cross sections are essentially determined by the longitudinal strength (or dynamical structure) functions $s^{31,41,42}$ in the charge (CDE) $S_{n n}(q, \omega)$ and the spin density channel (SDE) $S_{m m}(q, \omega)$ :

$$
\begin{gathered}
\frac{d^{2} \sigma^{C}}{d \omega_{s} d \Omega_{s}} \propto\left|\hat{\mathbf{e}}_{i} \cdot \hat{\mathbf{e}}_{s}\right|^{2} S_{n n}(q, \omega), \\
\frac{d^{2} \sigma^{S}}{d \omega_{s} d \Omega_{s}} \propto\left|\hat{\mathbf{e}}_{i} \times \hat{\mathbf{e}}_{s}\right|^{2} S_{m m}(q, \omega),
\end{gathered}
$$

where $\omega=\omega_{i}-\omega_{s}$ is the energy difference of the incoming and scattered photon. Using the above expressions one assumes that only conduction band electron are involved, and only off-resonance Raman peaks excited by laser energies above the valence-conduction band gap can be described.

To obtain $S_{n n}(q, \omega)$ and $S_{m m}(q, \omega)$ within the TDLSDFT, we have calculated the response to operators whose spatial dependence in the on-plane wave vector $\vec{q}$ is a plane wave $e^{i q \vec{r}}$. We refer the reader to Refs. 30 and 31 for a detailed description of the longitudinal response at $q \approx 0$, and at finite $q$.

Figure 6 shows the CDE's and SDE's at $B=0$ for the $N$ $=10$ ring and several $q$ values. For each $q$ value, we have considered in the expansion of the plane wave into Bessel functions of integer order

$$
e^{i \overrightarrow{q r}}=J_{0}(q r)+\sum_{L>0} i^{L} J_{L}(q r)\left(e^{i L \theta}+e^{-i L \theta}\right)
$$

as many multipole terms as needed to exhaust the $f$-sum rule $^{31}$ 


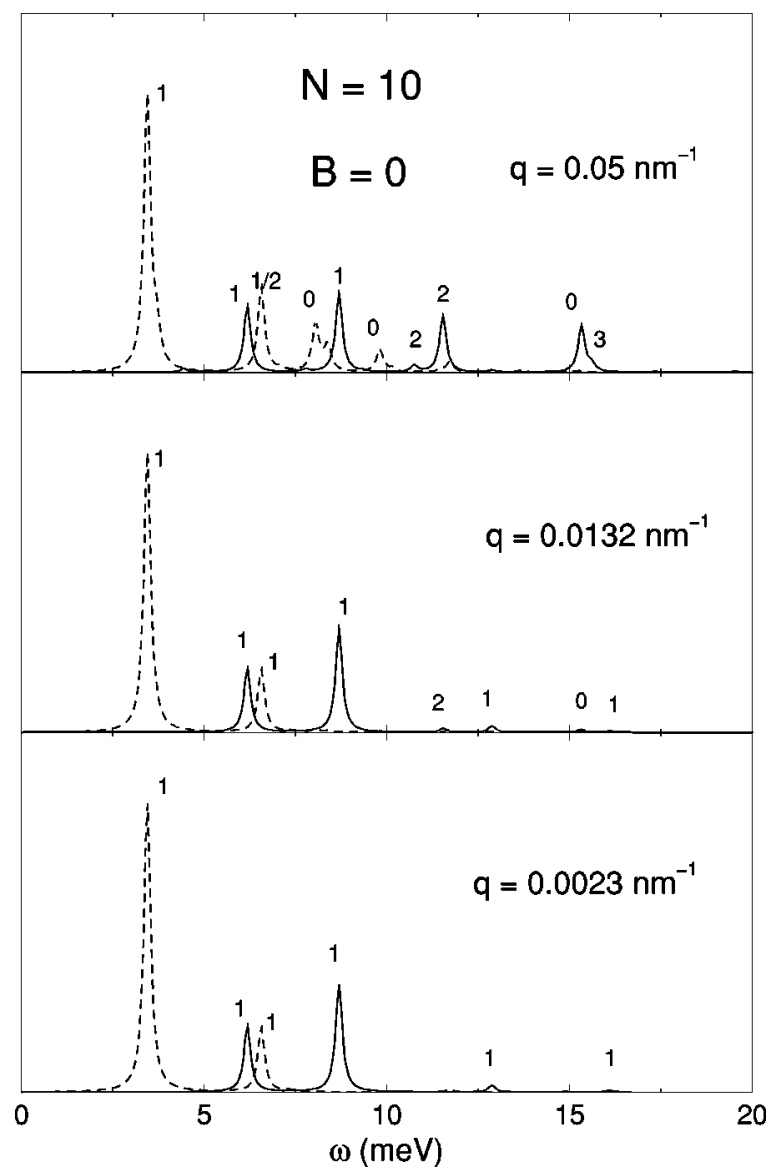

FIG. 6. Charge (solid lines) and spin density (dashed lines) strengths in arbitrary units as a function of $\omega$ for the $N=10$ ring at $B=0$ and several $q$ values. The multipolarity of the peaks is indicated.

$$
m_{1}^{(n n)}\left[e^{i \overrightarrow{q r}}\right]=m_{1}^{(m m)}\left[e^{i \overrightarrow{q r}}\right]=q^{2} \frac{N}{2}
$$

We infer from this figure that the modes have no appreciable wave-vector dispersion, a clear signature of the "zerodimensionality" of the ring. A similar conclusion was drawn for dots, ${ }^{31}$ in agreement with experiment. ${ }^{26}$ It can also be observed from this figure that a few number of multipoles (up to $L=2$ for the largest $q$ value) is enough to yield the plane wave response, and that in the $q \approx 0$ long wavelength limit only the dipole $L=1$ mode contributes.

The $B$ dispersion of the dipole mode for the $N=10$ ring in the long wavelength limit is shown in the top panel of Fig. 3. It is worth to see that edge modes have a smooth $B$ dispersion except in the regions where the ring is in the ferromagnetic $S_{z}=1$ phase. The discontinuities in the magnetoplasmon and spin modes, or equivalently these appearing in the absorption spectrum, ${ }^{11}$ are features that could be detected in optical-absorption experiments, making observable the ST and TS transitions and the ferromagnetic phases.

In the one-electron ring-wire case, the energies of the dipole modes are given by

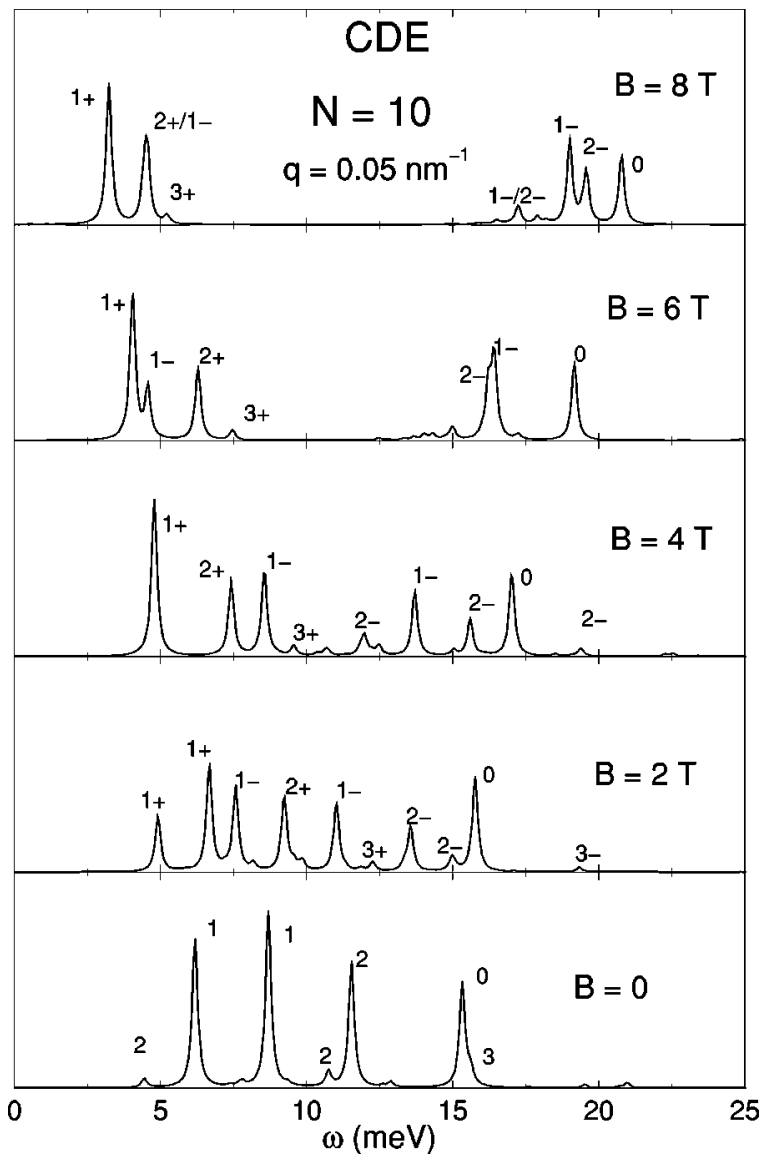

FIG. 7. Charge density strength in arbitrary units for $N=10, q$ $=0.05 \mathrm{~nm}^{-1}$ and different $B$ values. The multipolarity and polarization of the main peaks is indicated.

$$
\begin{gathered}
\omega_{+}=\epsilon_{l+1}-\epsilon_{l}=\frac{\hbar^{2}}{2 m^{*} R_{0}^{2}}\left[1-2\left(\frac{\Phi}{\Phi_{0}}-l\right)\right], \\
\omega_{-}=\epsilon_{l-1}-\epsilon_{l}=\frac{\hbar^{2}}{2 m^{*} R_{0}^{2}}\left[1+2\left(\frac{\Phi}{\Phi_{0}}-l\right)\right] .
\end{gathered}
$$

These excitation energies show $\mathrm{AB}$ oscillations with the same period as gs energy and persistent current oscillations. Furthermore, they are discontinuous at the $B$ values corresponding to level crossings. The $B$ dependence of the absorption spectrum of a quasi-ring-wire with two electrons was obtained $^{11}$ by an exact diagonalization calculation, and it was shown to display fractionary $\mathrm{AB}$ oscillations of period $\Phi_{0} / 2$. The ring-wire character of the system is crucial for it to present distinct $\mathrm{AB}$ oscillations.

Neither spin nor charge density modes with $L \neq 1$ can be detected in FIR spectroscopy. In contradistinction, Raman spectroscopy in QD's has proved to be able to disentangle spin from density modes, and to identify non-dipole charge and spin density modes. ${ }^{27}$ We show the charge and spin density strengths for $q=0.05 \mathrm{~nm}^{-1}$ in Figs. 7 and 8 , respectively. Only modes up to the octupole $(L=3)$ mode are excited with some intensity. The octupole strength is very small because at $q=0.05 \mathrm{~nm}^{-1}$ it contributes very little to the 


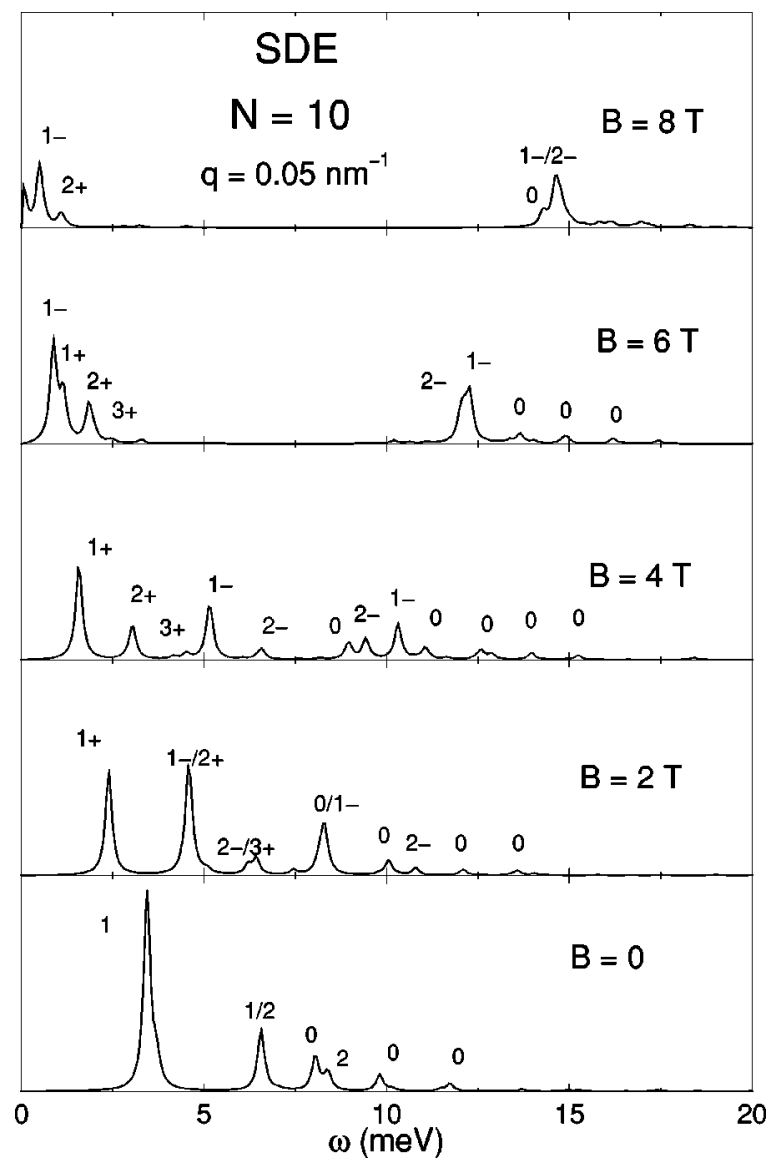

FIG. 8. Same as Fig. 7 for the spin density strength.

Bessel-function expansion of the plane wave in the region where the electronic density is concentrated, see Fig. 2. The monopole $L=0$ strength in the charge density channel has only one high-energy peak, whereas it is very fragmented in the spin density channel.

We have identified the polarization of the main peaks with $\mathrm{a}+(-)$ sign when the peak arise from $\Delta\left|L_{z}\right|=L(-L)$ changes in orbital angular momentum. They come, respectively, from the $e^{i L \theta}$ and $e^{-i L \theta}$ contributions in the expansion of the plane wave, Eq. (6). The lower energy $(+)$ peak corresponds to the outer edge mode, the high energy $(-)$ peak to the bulk mode, and the lower $(-)$ peak to the inner edge mode. ${ }^{15,21}$ Actually, the distinction between edge and bulk modes only makes sense at high enough magnetic fields; in the present case, well developed Landau bands appear at $B$ $\sim 5 \mathrm{~T}$, and for lower magnetic fields the charge and spin density spectrum is fairly complex.

We have collected in Figs. 9 and 10 the $B$ dispersion of the more intense CDE's and SDE's, respectively, for $q$ $=0.05 \mathrm{~nm}^{-1}$. It can be noted that the discontinuities in the energy of the inner edge dipole mode $(-)$ we have discussed in the long wavelength limit (top panel of Fig. 3) are well visible. For quadrupole modes, at some $B$ values we have found more than one single intense peak of a give polarization (see also Fig. 7). In these cases, the more intense peak is indicated by a full symbol, and the less intense peak by an open symbol.

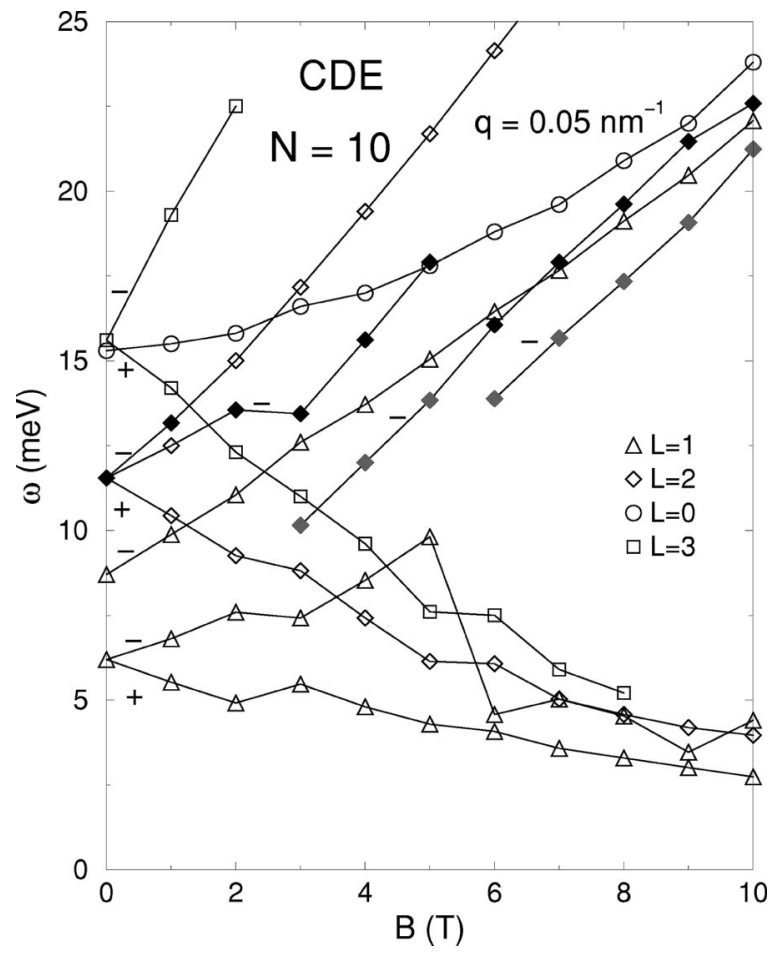

FIG. 9. $B$ dispersion of the more intense CDE's of the $N=10$ ring for $q=0.05 \mathrm{~nm}^{-1}$. The polarization of the peaks is indicated, and the lines have been drawn to guide the eye.

At high magnetic fields, the positive $B$ dispersion CDE's and SDE's have a clear tendency to bundle. These peaks arise from interband transitions whose Landau index differs in one unit. As explained in Ref. 31 for the case of dots, this

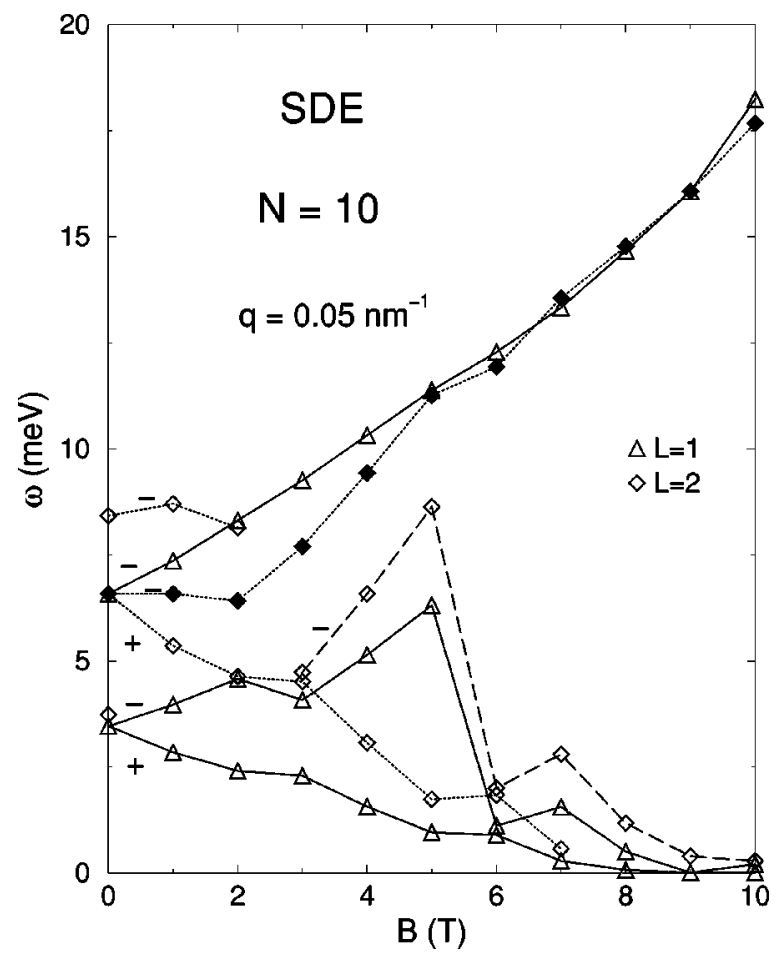

FIG. 10. Same as Fig. 9 for the more intense SDE's. 

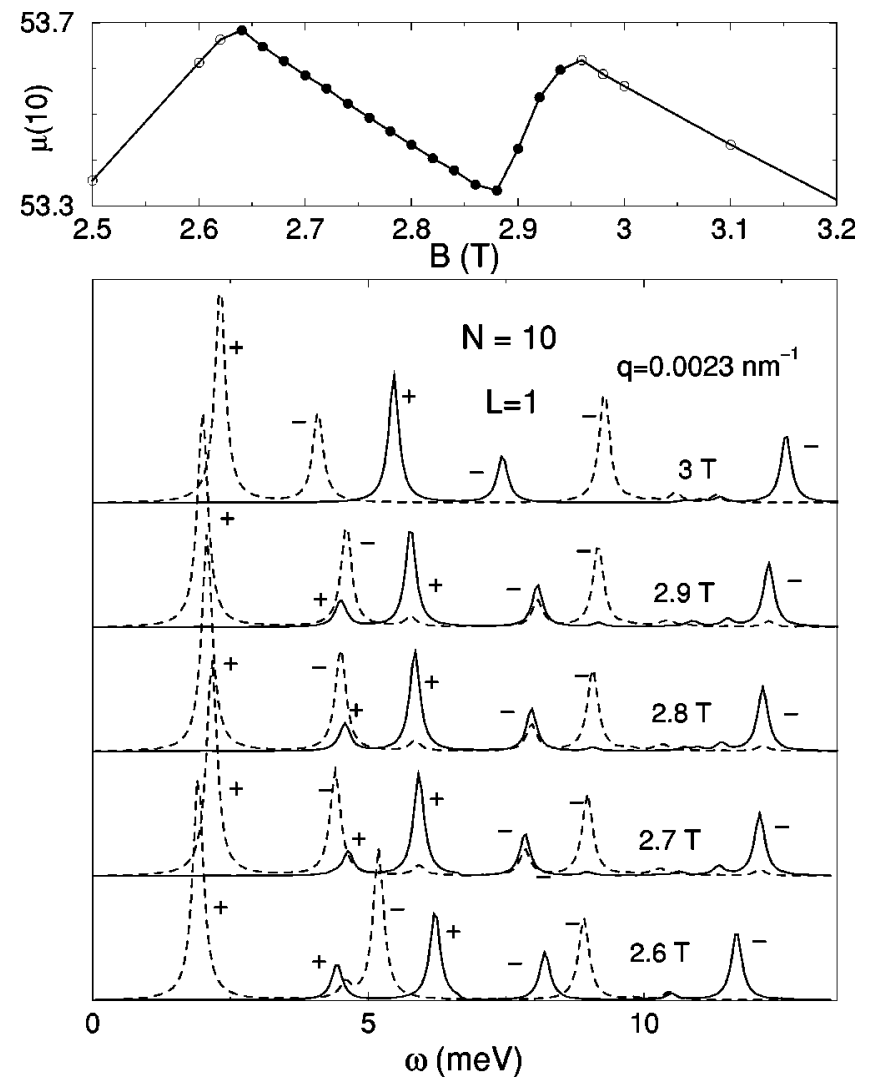

FIG. 11. Top panel: $\mu(10)(\mathrm{meV})$ around $B \sim 3 \mathrm{~T}$. The solid symbols represent the ferromagnetic phase, and the open symbols the paramagnetic phase. Bottom panel: Charge (solid lines) and spin density (dashed lines) dipole strengths in arbitrary units as a function of $\omega(\mathrm{meV})$ for the $N=10$ ring at $q=2.3 \times 10^{-3} \mathrm{~nm}^{-1}$ around $B \sim 3 \mathrm{~T}$.

happens because the Landau bands are made of many s.p. states with different $l$ values and energies rather $l$ independent if $B$ is large enough. Only finite-size effects and the $L$ dependence of the electron-hole interaction cause some dependence on the mode multipolarity. The origin of the high energy, weak quadrupole CDE's are excitations involving interband transitions whose Landau index differs in two units. Notice also that the quadrupole spin density strength is very fragmented, especially around the $B \sim 5 \mathrm{~T}$ ferromagnetic region.

It is interesting to notice that "- " edge modes only appear in the dipole case. Recalling that these are inner edge modes, Fig. 2 helps to understand this finding. It can be seen that only the $L=0$ and 1 multipoles of the plane wave expansion are sizeable in the inner edge region, and as a consequence, the strength of the $J_{L}(q r) e^{-i L \theta}$ component with $L>1$ goes to the high energy mode of the same polarization. In contradistinction, the "+" outer edge mode has no highenergy counterpart and takes the whole strength of the $J_{L}(q r) e^{i L \theta}$ component. The high sensitivity of the "- " edge modes to the ring morphology might help to shed light onto the actual structure of the ring inner edge. ${ }^{19}$ We recall that the experimental nanoscopic rings ${ }^{3}$ have been fabricated from InAs self-assembled dots grown on GaAs covered by a thin layer of GaAs by an annealing process.
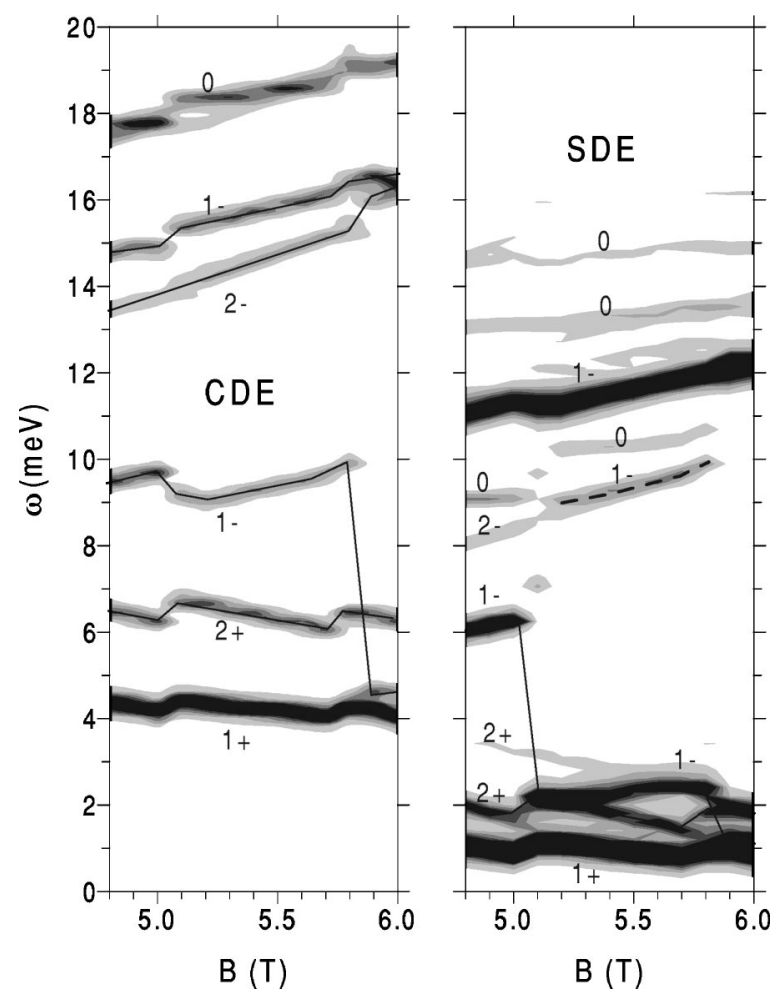

FIG. 12. Gray-scale plot of CDE's (left panel) and SDE's (right panel) of the $N=10$ ring for $q=0.05 \mathrm{~nm}^{-1}$ in the $B \sim 5-6 \mathrm{~T}$ region. The lines have been drawn to guide the eye. The dashed line along one of the $1^{-}$SDE's denotes an excitation branch arising from the coupling between charge and spin density responses.

The existence of ferromagnetic phases causes the coupling of spin and charge density responses. This effect can be detected experimentally, and as a matter of fact, it has been observed in QD's using Raman spectroscopy. ${ }^{27}$ This means that in the spin density channel, in addition to the main SDE's, a spin-dependent external field excites other low intensity peaks lying at the energy of the more intense CDE's which appear in the charge density channel, and conversely. The coupling of CDE's and SDE's has been thoroughly discussed within TDLSDTF in Refs. 30 and 42 for QD's, and in Ref. 43 for antidots. An example of how this coupling looks for rings is presented in Fig. 11, where we show the charge and spin dipole modes near $B \sim 3 \mathrm{~T}$. One can see that both responses are decoupled if the g.s. is paramagnetic, as it is at $B=2.6 \mathrm{~T}$, and are coupled if the gs is ferromagnetic, as it is at $B=2.8 \mathrm{~T}$, for example.

The existence of islands of spin-triplet gs's of rather wide $B$ extension is a characteristic of nanoscopic rings which mesoscopic rings hosting several thousand electrons lack of. Indeed, only for few electron rings the spin polarization $\xi$ $=\left(N_{\uparrow}-N_{\downarrow}\right) / N$ is sizeable in the triplet state, a $20 \%$ for the $N=10$ ring. Large couplings only arise from large spin polarizations through the spin dependence of the exchangecorrelation potential. ${ }^{30}$

Finally, we present in Fig. 12 a gray-scale plot of the Raman strength in the charge (left panel) and spin density channels (right panel) corresponding to the $4.8 \leqslant B \leqslant 6$ T region. This figure clearly reveals the ST and TS transition 
points as discontinuities in the $B$ dispersion of the multipole modes, especially visible in the inner edge dipole mode. These discontinuities are fingerprints of g.s. spin transitions and could be detected in Raman experiments.

\section{SUMMARY}

In this work we have studied physical aspects of charge and spin density responses of nanoscopic rings that might be detected by Raman spectroscopy. We have considered rings whose morphology is close to that of the systems recently fabricated, excluding for this reason the interesting case of nanoscopic ring wires that can also be addressed using the same method. We have taken as case of study a ring with 10 electrons and have seen that monopole to quadrupole modes clearly appear in the spin, and especially in the charge density channel. Both channels are coupled if the g.s. is ferromagnetic, and the ST and TS transition points may be identified as discontinuities in the $B$ dispersion of the multipole modes.
We have also discussed the addition spectrum of rings hosting up to 24 electrons, and have found that their shell structure is similar to that of quantum dots up to $N=6$. For larger $N$ values this is not so; the rings present shell closures at $N=2,6,10,16$, and 24. Hund's first rule is satisfied up the fourth shell for the assumed ring geometry.

The non-one-dimensional character of these rings hinders the existence of fractionary $\mathrm{AB}$ oscillations in the sp energy levels, permanent current and collective modes. Yet, the existence of triplet g.s.'s is manifested by the correlated presence of spin gaps in the $B$ dipersion of the sp levels, discontinuities in the multipole collective modes, and small amplitude oscillations superimposed to the integer $\mathrm{AB}$ oscillation of the persistent current and ring total energy.

\section{ACKNOWLEDGMENTS}

This work has been performed under Grants No. PB981247 from DGESIC, Spain, and 2000SGR00024 from Generalitat de Catalunya.
${ }^{1}$ J.M. Garcia et al., Appl. Phys. Lett. 71, 2014 (1997).

${ }^{2}$ A. Lorke and R.J. Luyken, Physica B 256, 424 (1998).

${ }^{3}$ A. Lorke, R.J. Luyken, A.O. Govorov, J.P. Kotthaus, J.M. Garcia, and P.M. Petroff, Phys. Rev. Lett. 84, 2223 (2000).

${ }^{4}$ C. Dahl, J.P. Kotthaus, H. Nickel, and W. Schlapp, Phys. Rev. B 48, 15480 (1993).

${ }^{5}$ C.R. Proetto, Phys. Rev. B 46, 16174 (1992).

${ }^{6}$ E. Zaremba, Phys. Rev. B 53, R10 512 (1996).

${ }^{7}$ T. Chakraborty and P. Pietiläinen, Phys. Rev. B 50, 8460 (1994).

${ }^{8}$ V. Gudmundsson and Á. Loftsdóttir, Phys. Rev. B 50, 17433 (1994).

${ }^{9}$ L. Wendler, V.M. Fomin, A.V. Chaplik, and A.O. Govorov, Phys. Rev. B 54, 4794 (1996).

${ }^{10}$ V. Halonen, P. Pietiläinen, and T. Chakraborty, Europhys. Lett. 33, 377 (1996).

${ }^{11}$ K. Niemelä, P. Pietiläinen, P. Hyvönen, and T. Chakraborty, Europhys. Lett. 36, 533 (1996).

${ }^{12}$ W.-C. Tan and J.C. Inkson, Phys. Rev. B 60, 5626 (1999).

${ }^{13}$ S. Viefers, P. Singha Deo, S.M. Reimann, M. Manninen, and M. Koskinen, Phys. Rev. B 62, 10668 (2000).

${ }^{14}$ I. Magnusdottir and V. Gudmundsson, Phys. Rev. B 60, 16591 (1999).

${ }^{15}$ A. Emperador, M. Barranco, E. Lipparini, M. Pi, and L1. Serra, Phys. Rev. B 59, 15301 (1999).

${ }^{16} \mathrm{H}$. Hu, J-L. Zhu, and J-J. Xiong, Phys. Rev. B 62, 16777 (2000).

${ }^{17}$ M. Koskinen, M. Manninen, B. Mottelson, and S.M. Reimann, Phys. Rev. B 63, 205323 (2001).

${ }^{18}$ P. Borrmann and J. Harting, Phys. Rev. Lett. 86, 3120 (2001).

${ }^{19}$ A. Puente and L1. Serra, Phys. Rev. B 63, 125334 (2001).

${ }^{20}$ D. Mailly, C. Chapelier, and A. Benoit, Phys. Rev. Lett. 70, 2020 (1993).

${ }^{21}$ A. Emperador, M. Pi, M. Barranco, and A. Lorke, Phys. Rev. B 62, 4573 (2000).

${ }^{22}$ P.L. McEuen, E.B. Foxman, U. Meirav, M.A. Kastner, Y. Meir,
N.S. Wingreen, and S.J. Wind, Phys. Rev. Lett. 66, 1926 (1991).

${ }^{23}$ R.C. Ashoori, H.L. Stormer, J.S. Weiner, L.N. Pfeiffer, and K.W. West, Phys. Rev. Lett. 68, 3088 (1992); 71, 613 (1993).

${ }^{24}$ S. Tarucha, D.G. Austing, T. Honda, R.J. van der Hage, and L.P. Kouvenhoven, Phys. Rev. Lett. 77, 3613 (1996).

${ }^{25}$ D.J. Lockwood, P. Hawrylak, P.D. Wang, C.M. Sotomayor Torres, A. Pinczuk, and B.S. Dennis, Phys. Rev. Lett. 77, 354 (1996).

${ }^{26}$ C. Schüller, G. Biese, K. Keller, C. Steinebach, D. Heitmann, P. Grambow, and K. Eberl, Phys. Rev. B 54, R17 304 (1996).

${ }^{27}$ C. Schüller, K. Keller, G. Biese, E. Ulrichs, L. Rolf, C. Steinebach, D. Heitmann, and K. Eberl, Phys. Rev. Lett. 80, 2673 (1998).

${ }^{28}$ M. Ferconi and G. Vignale, Phys. Rev. B 50, 14722 (1994).

${ }^{29}$ M. Pi, M. Barranco, A. Emperador, E. Lipparini, and Ll. Serra, Phys. Rev. B 57, 14783 (1998).

${ }^{30}$ Ll. Serra, M. Barranco, A. Emperador, M. Pi, and E. Lipparini, Phys. Rev. B 59, 15290 (1999).

${ }^{31}$ M. Barranco, L. Colletti, E. Lipparini, A. Emperador, M. Pi, and L1. Serra, Phys. Rev. B 61, 8289 (2000).

${ }^{32}$ M. Fricke, A. Lorke, J.P. Kotthaus, G. Medeiros-Ribeiro, and P.M. Petroff, Europhys. Lett. 36, 197 (1996).

${ }^{33}$ A. Lorke, M. Fricke, B.T. Miller, M. Haslinger, J.P. Kotthaus, G. Medeiros-Ribeiro, and P.M. Petroff, Inst. Phys. Conf. Ser. 155, 803 (1996).

${ }^{34}$ B.T. Miller, W. Hansen, S. Manus, R.J. Luyken, A. Lorke, and J.P. Kotthaus, Phys. Rev. B 56, 6764 (1997).

${ }^{35}$ O. Steffens, M. Suhrke, and U. Rössler, Europhys. Lett. 44, 222 (1998).

${ }^{36}$ S. Tarucha, D.G. Austing, Y. Tokura, W.G. van den Wiel, and L.P. Kouvenhoven, Phys. Rev. Lett. 84, 2485 (2000).

${ }^{37}$ G. Vignale and M. Rasolt, Phys. Rev. Lett. 59, 2360 (1987); Phys. Rev. B 37, 10685 (1988).

${ }^{38}$ M. Moskalets, Physica B 291, 350 (2000).

${ }^{39}$ A. Pinczuk and G. Abstreiter, Light Scattering in Solids V, edited 
by M. Cardona and G. Guntherodt, Vol. 66 of Topics in Applied Physics (Springer-Verlag, Berlin, 1989), p. 153.

${ }^{40}$ C. Steinebach, C. Schüller, and D. Heitmann, Phys. Rev. B 59, 10240 (1999).
${ }^{41}$ F.A. Blum, Phys. Rev. B 1, 1125 (1970).

${ }^{42}$ O. Steffens and M. Suhrke, Phys. Rev. Lett. 82, 3891 (1999).

${ }^{43}$ A. Emperador, M. Pi, M. Barranco, E. Lipparini, and Ll. Serra, Jpn. J. Appl. Phys. 40, 518 (2001). 